\title{
Estudo Comparativo das Tendências de e-Learning e das Características de Soluções Líderes de Learning Management System
}

Maria Helena Mauro, Programa de Pós-graduação em Informática e Gestão do Conhecimento da Universidade Nove de Julho, mahelenamauro@gmail.com Marcos Antonio Gaspar, Programa de Pós-graduação em Informática e Gestão do Conhecimento da Universidade Nove de Julho, marcos.antonio@uni9.pro.br Fábio Kazuo Ohashi, Programa de Pós-graduação em Informática e Gestão do Conhecimento da Universidade Nove de Julho, kazuo@uni9.pro.br Fabio Luis Falchi de Magalhães, Programa de Pós-graduação em Informática e Gestão do Conhecimento da Universidade Nove de Julho, fabiosimp@gmail.com

\section{Resumo}

Esta pesquisa busca analisar soluções de gestão de aprendizagem (LMS - Learning Management System) disponíveis no mercado corporativo, confrontando suas características com as tendências apontadas na literatura a respeito da evolução do elearning. Para tanto, a pesquisa exploratória-qualitativa realizada avaliou as aplicações de três empresas fornecedoras consideradas líderes de mercado pela consultoria Gartner no segmento LMS no período 2013-2017. A Gartner publica estudo anual com o intuito de avaliar quais características e recursos destes softwares contribuem para o posicionamento da solução como líder nesse mercado. Os resultados verificados na pesquisa indicam que a integração com outros sistemas de Recursos Humanos contribui para o gerenciamento dos funcionários, bem como para seu desempenho e plano de carreira. Em complemento, verificou-se ainda que a inclusão de ferramentas e funcionalidades de redes sociais aumenta o interesse dos funcionários na utilização da solução LMS, incentivando a transferência de conhecimento entre eles. Como conclusão, verificou-se que as características encontradas nas soluções LMS desenvolvidas pelas empresas líderes de mercado guardam estreita relação com as características levantadas na literatura sobre e-learning, exceção feita à tendência de gamificação abordada pela literatura, que não foi verificada com destaque nas soluções LMS analisadas.

Palavras-chave: e-learning, educação à distância, educação corporativa, software de gestão da aprendizagem, gestão do conhecimento.

\section{A Comparative Study of e-Learning Trends and Characteristics of Leading Learning Management System Solutions}

\begin{abstract}
This research aims to analyze LMS solutions (Learning Management System) available in the corporate market, confronting its features with the trends identified in the literature regarding the evolution of e-learning. In order to achieve this goal, a qualitative exploratory research was carried out evaluating the applications of three software suppliers considered market leaders by Gartner Consulting in the LMS reports in the 2013-2017 period. Gartner publishes annual study in order to assess which features and capabilities of these software help to establish the solution as a leader in this market. The results indicate that the integration with other Human Resources systems contributes to manage the employees' performance and their career plan. In addition, the inclusion of networking tools and features increases the interest of employees to apply the LMS solution, as well as encourages the knowledge transfer
\end{abstract}


among them. As a conclusion, it is possible to state that the LMS characteristics found in the analyzed solutions developed by market leading companies keep close relationship with raised features verified in literature about e-learning, except the trend of gamification addressed by literature, which has not been verified in the LMS solutions analyzed.

Key words: e-learning, distance learning, corporate education, learning management system, knowledge management.

\section{INTRODUÇÃO}

Gerenciar os talentos da empresa é um assunto que tem se tornando muito importante para as áreas de Gestão de Pessoas na atualidade (Venkateswaran, 2012; Fuller 2013). Para muitas empresas, as pessoas são um dos recursos mais valiosos, além de ser um dos principais elementos na construção do conhecimento organizacional (Cuellar; Aguilar, 2012). Conforme afirma Venkateswaran (2012), gerenciar pessoas e seus talentos não é simplesmente atrair os melhores profissionais, mas sim cuidar de sua contratação, desenvolvimento, retenção e promoção. Assim, é possível observar a importância cada vez maior do desenvolvimento dos funcionários e, consequentemente, do papel da educação corporativa como forma de desenvolver os talentos dos profissionais da empresa (Castro; Eboli, 2013).

Assim, observa-se que estudos referentes à aprendizagem organizacional, como demonstram Igarashi et al. (2009), buscam gerar subsídios para as organizações identificarem elementos que apoiem a diferenciação em prol da construção de vantagens competitivas à organização. Nesse sentido, um dos elementos que favorece $\mathrm{o}$ estabelecimento da diferenciação é a Tecnologia da Informação (TI). A TI pode ser empregada para a transferência de conhecimentos, para a diminuição de barreiras de comunicação, para a promoção de interação entre as pessoas e ainda para o melhor gerenciamento do conhecimento na empresa (Fernandes; Abreu, 2014).

A TI tem ganhado importância cada vez maior como indutora e viabilizadora de ações voltadas à educação corporativa e, consequentemente, no desenvolvimento dos talentos na organização. Para Oliveira et al. (2016), a TI é que permite o desenvolvimento da educação à distância e fornece condições para a organização trabalhar com processos novos. Na visão de Gurunath e Kumar (2015), as tecnologias são um avanço no processo de ensino aprendizagem e a cada ano surgem novos aplicativos para reduzir custos, aumentar o acesso e atender as necessidades de ensino nas corporações.

Considerando-se tal contexto, uma das ferramentas de TI mais utilizadas no processo de aprendizagem nas empresas é o LMS (Learning Management System), que é uma solução empregada principalmente na educação à distância praticada pelas empresas. Para Fuller (2013, p. 26), "o acrônimo LMS refere-se a um conjunto de funcionalidades projetadas para gerenciar o conteúdo de aprendizagem, o progresso do aluno e as interações dos alunos". Porém, um software LMS envolve muito mais que simplesmente um sistema de aprendizagem.

Em função disto, esta pesquisa tem como objetivo analisar soluções de gestão de aprendizagem (LMS - Learning Management System) disponíveis no mercado corporativo, confrontando suas características com as tendências apontadas na literatura a respeito da evolução do e-learning. Para a realização da análise indicada utilizou-se pesquisa bibliográfica sobre as tendências demonstradas e caminhos apontados nas pesquisas acadêmicas sobre e-learning, comparando-se tais características com as funcionalidades ofertadas pelas empresas fornecedoras de soluções LMS consideradas 
líderes de mercado. Para tanto, o Relatório Gartner foi utilizado com informações de pesquisas de mercado que apontam as três empresas líderes no segmento mencionado.

Foi utilizada como base para a escolha das ferramentas, as aplicações de empresas líderes de mercado identificadas por pesquisas realizadas nos cinco últimos anos pela Gartner (2013, 2014, 2015 e 2017). A pesquisa utilizada foi o Relatório Magic Quadrant for Talent Management Suites.

\section{FUNDAMENTAÇÃO TEÓRICA}

\subsection{E-learning e learning management system}

O LMS (Learning Management System) tem se tornando uma das principais ferramentas de desenvolvimento on-line utilizadas pelo mercado corporativo para a promoção da aprendizagem de profissionais nas empresas. Para Fuller (2013), o LMS é um conjunto de funcionalidades utilizado para gerenciar o conteúdo de aprendizagem, avaliar o progresso do aluno e facilitar as interações entre os alunos. Segundo Clementino (2008), as soluções LMS são utilizadas para o gerenciamento de situações de aprendizagem nas empresas, notadamente em função da possibilidade de integrar múltiplas mídias, recursos e linguagens em prol do processo de aprendizagem.

Nos últimos anos, as soluções LMS têm avançado bastante, buscando adaptar-se às necessidades do processo de aprendizagem desenvolvido no ambiente corporativo. Assim, as soluções LMS têm englobado novas funcionalidades para atender as constantes mudanças ocorridas no contexto corporativo (Peña et al., 2012). Segundo Cohen (2010), o LMS está sendo constantemente reinventado, sendo sua atualização voltada à inclusão de ferramentas de colaboração que capturam a aprendizagem e incentivam a colaboração interna na organização. Além disso, a gestão da aprendizagem evoluiu, facilitando assim o compartilhamento de informações e a colaboração dos funcionários. Isto inclui treinamentos formais e informais, além da aprendizagem em toda a empresa. As soluções LMS atuais incorporaram funcionalidades presentes nas redes sociais, que promovem maior interação e trocas de informações e conhecimentos pelos envolvidos.

Kane, Combre e Berge (2010, p. 66) também observam que "este movimento para vincular as ferramentas de redes sociais ao local de trabalho está crescendo". A tendência de escritórios virtuais separou funcionários e reduziu a partilha informal de conhecimentos face a face. Tal desafio significa que as empresas devem encontrar meios para que os funcionários compartilhem conhecimentos. Nesse sentido, as atuais soluções LMS vinculam ferramentas de aprendizagem às ferramentas de redes sociais, o que promove a reunião dos colaboradores geograficamente dispersos para a promoção de transferência de conhecimentos entre si. Esta tendência de vincular as ferramentas sociais aos softwares de aprendizagem foi também identificada por autores que pesquisam as soluções de e-learning em instituições de ensino (Silva; Brito, 2014; Ylmaz, 2016).

Fuller (2013) argumenta que o LMS evoluiu para se tornar o ponto focal de integração com outros sistemas de aprendizagem da organização. Assim, a essência do conceito de integração de outros elementos ao LMS se faz uma realidade na atualidade do ambiente corporativo. Um dos modelos propostos é de integração de conhecimentos, desempenhos e aprendizagens, que atua como a base para a formação de um sistema de conhecimentos, performance e gestão de aprendizagem organizacional.

Gurunath e Kumar (2015) estudaram outra tendência de aplicação no LMS, que é a computação em nuvem (cloud computing). Segundo os autores, as empresas já estão 
ofertando o LMS como SaaS (Software as a Service - software como serviço). Assim, o LMS baseado em computação em nuvem surge como opção para as empresas. Como o LMS é armazenado em nuvem, não é necessário o gerenciamento do software. Outra tendência apontada por Gurunath e Kumar (2015) é que o LMS passou a oferecer interface mais moderna para os usuários.

Bezhovski e Poorani (2016) também citam como tendência para o e-learning a utilização do $S a a S$, bem como a gamificação e o blended learning (aprendizagem mesclada ou híbrida) que mistura práticas do ensino presencial e à distância com o objetivo de melhorar o desempenho do aluno em ambas as modalidades de ensino.

\subsection{Relatórios Gartner}

Para o levantamento de tendências no mercado de soluções $e$-learning, foram utilizadas as pesquisas realizadas nos últimos cinco anos pela conceituada empresa Gartner de pesquisa de mercado. Os fornecedores analisados na pesquisa comercializam o LMS como módulos de uma solução mais ampla. Neste caso, o LMS apresenta-se como parte do Talent Management Suite.

Segundo a Gartner (2017), as soluções Talent Management (TM) suítes são compostas por um conjunto integrado de aplicativos que suporta a necessidade de a organização planejar, atrair, desenvolver, recompensar, envolver e reter talentos profissionais. Os componentes típicos de uma solução TM são: a) Planejamento de força de trabalho; b) Recrutamento e seleção; c) Gerenciamento de desempenho e objetivos; d) Gestão da aprendizagem; e) Planejamento de carreira e sucessão e, por fim, f) Gestão de recompensas.

Considerando-se o período analisado, três empresas aparecem como líderes nos relatórios pesquisados no período 2013 a 2017 (GARTNER, 2013, 2014, 2015 e 2017). Assim, foram analisadas as seguintes empresas provedoras de soluções TM/LMS: Cornerstone, SAP e Oracle. A Figura 1 apresenta a evolução da classificação dos fornecedores dessas soluções no período analisado. 


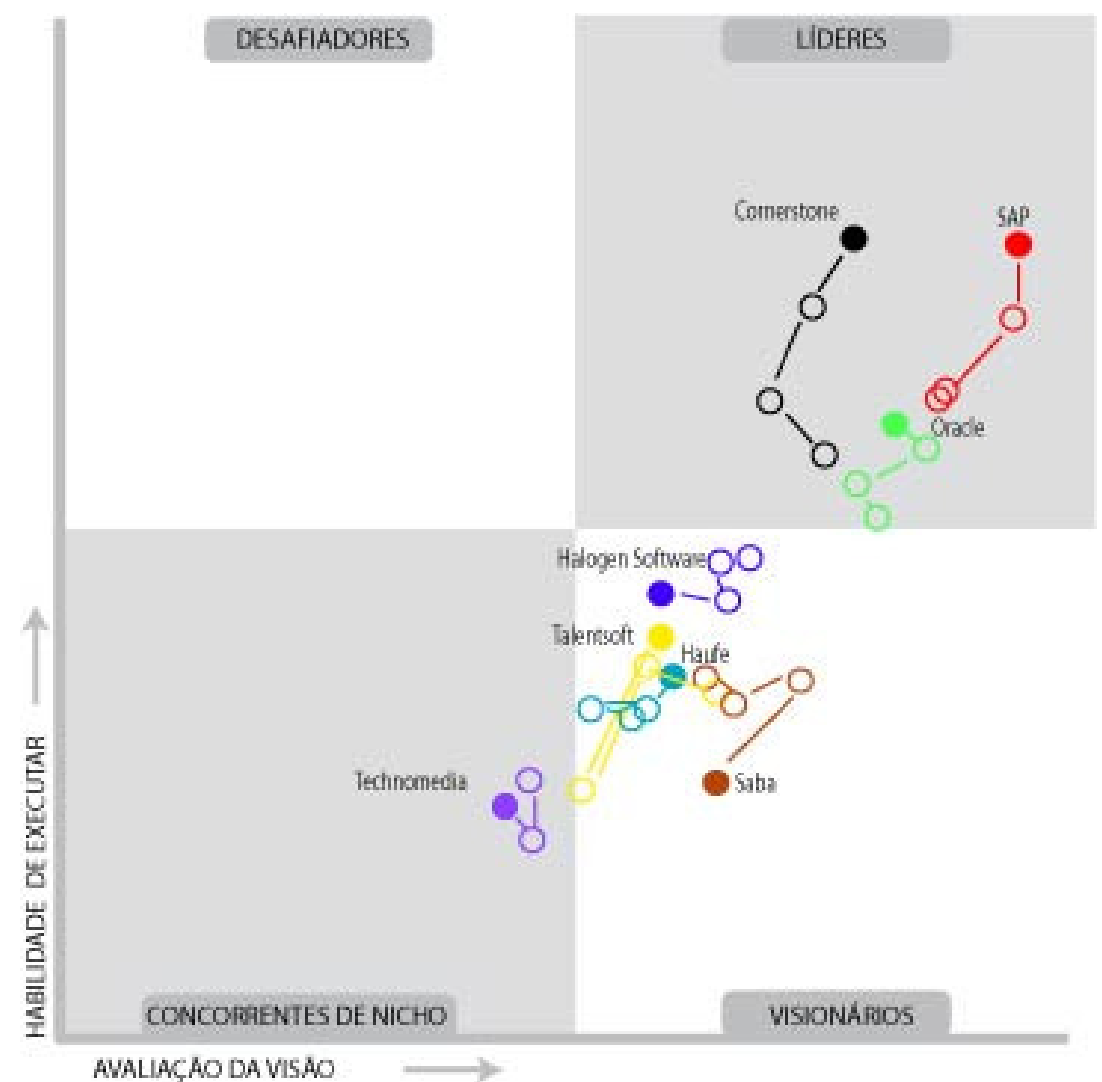

Figura 1: Evolução da classificação das empresas líderes de soluções LMS Fonte: elaborado pelos autores a partir de Gartner (2013, 2014, 2015, 2017).

O Relatório Magic Quadrant desenvolvido pela consultoria Gartner é uma representação gráfica do mercado de soluções de TI num determinado período. A figura anterior indica como as empresas fornecedoras de soluções em TI estão posicionadas a partir de critérios aplicáveis para um determinado mercado específico, como é o caso do software LMS no contexto desta pesquisa.

A matriz apresentada na Figura 1 é composta por dois eixos. O eixo horizontal apresenta a avaliação da visão da empresa fornecedora de soluções em termos de inovação tecnológica e abrangência de atendimento às necessidades do mercado. Em complemento, o eixo vertical apresenta avaliação do quanto as empresas fornecedoras de soluções têm a habilidade de executar e implementar o que prometem.

Assim, a matriz formada é composta por quatro quadrantes de classificação das empresas provedoras de solução, a saber: a) Líderes, por apresentarem a melhor visão tecnológica, com portfolio mais completo e ampla capacidade de executar o que prometem; b) Desafiadores, que são concorrentes importantes de mercado, com boa participação de mercado proporcionado pela boa capacidade de execução, porém atrás dos líderes em termos de abrangência e inovação; c) Visionários, que são empresas que têm aspectos fortes de inovação e visão abrangente de mercado, mas nas quais suas ferramentas ainda não conseguem entregar o que propõem e, por fim; d) Concorrentes de nicho, que são empresas que atendem nichos de mercado mais específicos. Elas não oferecem soluções que atendam qualquer empresa, mas provavelmente em seus segmentos suas soluções são consistentes.

\subsection{Relatórios Gartner sobre TM/LMS líderes de mercado}


Para a comparação proposta nesta pesquisa, considerou-se principalmente o componente de Gestão de Aprendizagem das soluções Talent Management Suítes pesquisadas pela Gartner em seus relatórios, uma vez que as funcionalidades LMS estão enquadradas principalmente neste tipo de solução. Para a Gartner (2013, 2014, 2015 e 2017), o módulo de gestão de aprendizagem inclui ferramentas para desenvolver, oferecer e acompanhar a aprendizagem, criar conteúdos, gerir competências e desenvolver competências, além de participação em cenários de aprendizagem social.

As principais características das soluções LMS disponibilizadas pelas três empresas classificadas como líderes de mercado são apresentadas a seguir.

Cornerstone - O conjunto TM da Cornerstone possui configuração robusta e a melhor relação aprendizado e desempenho em sua classe. A Cornerstone oferece todos os seis aplicativos componentes de soluções TM, sendo fornecido através de hospedagem em nuvem (cloud computing). Quanto à gestão de aprendizagem em especial, esta solução LMS da Cornerstone é considerada robusta quanto às funcionalidades específicas voltadas à aprendizagem; contando com um bom suporte móvel; fazendo uso de mecânica social e de jogos para envolver os funcionários (GARTNER, 2017).

Oracle - Oracle Talent Management Cloud é um subconjunto do Oracle Human Capital Cloud Management (HCM). As funcionalidades oferecidas abrangem o recrutamento, onboarding, desempenho, compensação, planejamento de carreira e gerenciamento de sucessão, além de aprendizado e planejamento de força de trabalho. O Oracle Talent Management Cloud é especialmente robusto em recrutamento, compensação e planejamento de sucessão. Considerando-se o módulo de gestão de aprendizagem, apresenta como pontos importantes a unificação de análises e relatórios, soluções mobile para dispositivos móveis, interação social e uma nova plataforma de aprendizagem social baseada em vídeos (GARTNER, 2017).

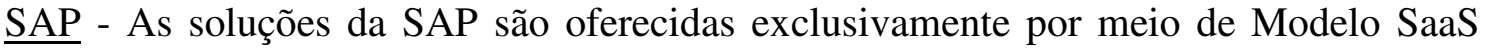
(Service as Assesment). A maioria dos clientes usa o gerenciamento de desempenho e o módulo de aprendizagem e recrutamento, mas a adoção dos outros módulos TM pelas empresas tem aumentado. O SAP SuccessFactors oferece soluções líderes em desempenho e planejamento de força de trabalho. A gestão de aprendizagem e planejamento da força de trabalho são especialmente robustas, sendo consideradas fáceis de utilizar tanto para funcionários, como para gestores (GARTNER, 2017).

No caso específico de soluções LMS, alguns pontos em comum foram evidenciados nos softwares analisados, tais como a integração com outros sistemas e a incorporação de ferramentas de interação social, dentre outros que serão apresentados mais adiante na análise promovida. Estas foram duas das características importantes dessas ferramentas destacadas nas três empresas listadas como líderes no mercado de soluções LMS.

\section{MATERIAIS E MÉTODOS DE PESQUISA}

Esta é uma pesquisa exploratório-qualitativa (GIL, 1999; GODOY, 1995) que busca adquirir conhecimento acerca da utilização de soluções LMS nas empresas, bem como compreender como tais soluções LMS estão evoluindo no mercado organizacional.

$\mathrm{Na}$ primeira fase foi realizado levantamento bibliográfico das tendências do mercado LMS observadas em artigos publicados nos últimos oito anos. Após esta análise, foi realizada a análise das empresas classificadas como líderes pela pesquisa da Gartner no 
período de 2013 a 2017. O Gartner Inc. é uma firma baseada em Stanford (EUA). O Relatório Quadrante Mágico oferece às empresas que pretendem adquirir soluções de TI resumos visuais para a avaliação dos fornecedores de soluções. Tal relatório classifica os fornecedores de software e serviços de TI, auxiliando as empresas compradoras a melhor avaliar as soluções disponíveis, contribuindo assim para a tomada de decisão sobre investimentos em TI por parte das empresas adquirentes.

O relatório Gartner também analisa as tendências do mercado para as ferramentas enfocadas, assim como seus pontos fortes e as precauções para cada fornecedora analisada. A Gartner realiza sua pesquisa considerando todos os módulos de TM descritos anteriormente (tópico 2.2). No entanto, para fins desta pesquisa foi dada ênfase nas características do módulo 'gestão da aprendizagem' dos TM suítes líderes de mercado (SAP, Oracle e Cornerstone).

\section{APRESENTAÇÃO E ANÁLISE DOS RESULTADOS}

Para a primeira etapa da pesquisa, voltada à análise das tendências apontadas na literatura sobre a evolução de soluções LMS, foram analisados artigos que abordaram as tendências dos LMS. Assim, foram considerados alguns autores que abordam o LMS no âmbito educacional, mas que analisam as ferramentas de forma mais abrangente. $\mathrm{O}$ Quadro 1 resume as principais tendências apontadas pelos autores identificados na pesquisa bibliográfica executada.

\begin{tabular}{l|l}
\hline \multicolumn{1}{c|}{ Autores } & \multicolumn{1}{c}{ Tendências apontadas } \\
\hline Kane, Combre e Berge (2009) & - Inclusão de ferramentas de redes sociais \\
& - Transferência de conhecimento \\
\hline Cohen (2010) & - Inclusão de ferramentas de colaboração \\
& - Funcionalidades presentes em redes sociais \\
& - Melhora da gestão da aprendizagem \\
\hline Fuller (2013) & - Integração com outros sistemas \\
\hline Silva e Brito (2014) & - Inclusão de ferramentas de redes sociais \\
\hline Gurunath e Kumar (2015) & - Utilização da computação em nuvem \\
& - SaaS (Software as a service) \\
\hline Bezhovski e Poorani (2016) & - Interface para o usuário mais moderna \\
\hline Ylmaz (2016) & - GaaS (Software as a service \\
\hline
\end{tabular}

Quadro 1: Tendências de evolução das soluções LMS apontadas por pesquisadores do tema

Fonte: elaborado pelos autores.

Para o desenvolvimento da visão de mercado foram considerados os Relatórios Gartner do período indicado (2013-2017). As soluções LMS estão enquadradas nas ferramentas de gestão de aprendizagem, que no Relatório Gartner incluem ferramentas para desenvolver, oferecer e acompanhar a aprendizagem, além de criar conteúdos, gerir desenvolver competências, bem como promover a participação em cenários de aprendizagem social.

O Quadro 2 apresenta as características e tendências verificadas nas soluções das três empresas líderes consideradas, conforme apontado na análise dos pontos fortes de cada empresa fornecedora de LMS considerada (Cornerstone OnDemand, SAP Success Factors e Oracle Talent Management). Também foram considerados os relatórios da Consultoria Gartner sobre o mercado de soluções LMS em geral. 


\begin{tabular}{|c|c|}
\hline $\begin{array}{c}\text { Soluções LMS líderes } \\
\text { analisadas }\end{array}$ & Relatório Gartner \\
\hline $\begin{array}{l}\text { Análise geral da } \\
\text { Gartner }\end{array}$ & $\begin{array}{l}\text { - Observado aumento da integração entre os módulos no período avaliado } \\
\text { - Melhorias na geração de relatórios }\end{array}$ \\
\hline $\begin{array}{l}\text { Análise da } \\
\text { Cornerstone } \\
\text { OnDemand }\end{array}$ & $\begin{array}{l}\text { - Considerado o melhor em sua classe de aprendizagem e desempenho } \\
\text { - Forte suporte móvel } \\
\text { - Bom uso de colaboração social e jogos para envolver os funcionários } \\
\text { - Disponibilidade somente como SaaS (Software as a service) }\end{array}$ \\
\hline $\begin{array}{l}\text { Análise da SAP } \\
\text { Success Factors }\end{array}$ & $\begin{array}{l}\text { - Soluções líderes de desempenho, em aprendizagem e planejamento da força } \\
\text { de trabalho } \\
\text { - Facilidade de uso para funcionários e gestores } \\
\text { - Disponibilidade também em SaaS (Software as a service) }\end{array}$ \\
\hline $\begin{array}{l}\text { Análise da Oracle } \\
\text { Talent Management }\end{array}$ & $\begin{array}{l}\text { - Unificação de análises e relatórios } \\
\text { - Forte integração com outros sistemas (como soluções de } e \text {-business) } \\
\text { - Suporte Mobile } \\
\text { - Interação social } \\
\text { - Nova plataforma de aprendizagem social baseada em vídeo }\end{array}$ \\
\hline
\end{tabular}

Quadro 2: Características e tendências das empresas líderes no mercado de soluções LMS

Fonte: elaborado pelos autores com base nos relatórios Gartner (2013, 2014, 2015 e 2017).

O Quadro 3 expõe um comparativo entre as principais características e tendências indicadas pela literatura considerada em relação aos pontos fortes verificados na análise das soluções LMS pesquisadas.

\begin{tabular}{|c|c|}
\hline $\begin{array}{c}\text { Principais tendências de evolução apontadas } \\
\text { na pesquisa bibliográfica }\end{array}$ & Relatório Gartner \\
\hline $\begin{array}{l}\text { - Vinculação de ferramentas de aprendizagem às } \\
\text { ferramentas de redes sociais (Kane; Combre; } \\
\text { Berge, 2009; Silva; Brito, 2014; Ylmaz, 2016) }\end{array}$ & $\begin{array}{l}\text { - Observado aumento da integração entre os } \\
\text { módulos no período avaliado } \\
\text { - Melhorias na geração de relatórios } \\
\text { - Evolução em aprendizagem e desempenho }\end{array}$ \\
\hline $\begin{array}{l}\text { - Inclusão de ferramentas de colaboração. } \\
\text { (Cohen, 2010) }\end{array}$ & $\begin{array}{l}\text { - Forte suporte móvel } \\
\text { - Bom uso de colaboração social e jogos para } \\
\text { envolver os funcionários }\end{array}$ \\
\hline $\begin{array}{l}\text { Ponto focal de integração com outros sistemas } \\
\text { de aprendizagem da organização (Fuller, } \\
\text { 2013) }\end{array}$ & $\begin{array}{l}\text { - Disponibilidade somente como SaaS (Software as } \\
\text { a service) } \\
\text { - Desempenho, em aprendizagem e planejamento } \\
\text { da força de trabalho }\end{array}$ \\
\hline $\begin{array}{l}\text { - Utilização da computação em nuvem SaaS } \\
\text { (Software as a service) } \\
\text { - Interface para o usuário mais moderna } \\
\text { (Gurunath; Kumar, 2015) }\end{array}$ & $\begin{array}{l}\text { - Facilidade de uso para funcionários e gestores } \\
\text { - Disponibilidade também em SaaS (Software as a } \\
\text { service) } \\
\text { - Unificação de análises e relatórios } \\
\text { - Forte integração com outros sistemas (como }\end{array}$ \\
\hline $\begin{array}{l}\text { - SaaS (Software as a service) } \\
\text { - Gamificação } \\
\text { - Blended learning (aprendizagem híbrida) } \\
\quad \text { (Bezhovski; Poorani, 2016) }\end{array}$ & $\begin{array}{l}\text { soluções } e \text {-business) } \\
\text { - Suporte mobile } \\
\text { - Interação social } \\
\text { - Nova plataforma de aprendizagem social baseada } \\
\text { em vídeo }\end{array}$ \\
\hline
\end{tabular}

Quadro 3: Tendências de evolução das soluções LMS versus pontos fortes das empresas líderes no mercado de soluções LMS

Fonte: elaborado pelos autores.

\section{CONCLUSÕES}

As pessoas estão sendo consideradas pelas empresas como um dos principais recursos na construção do conhecimento organizacional visando a criação de vantagens competitivas frente aos concorrentes. Por isso, gerenciar talentos está se tornando cada vez mais importante às empresas contemporâneas que desejam desenvolver diferenciais 
competitivos. Assim, ações voltadas à aprendizagem organizacional, dentre elas as ferramentas de e-learning, têm sido valorizadas atualmente nas empresas.

Esta pesquisa buscou apresentar uma análise de soluções de gestão de aprendizagem (LMS - Learning Management System) utilizadas no mercado corporativo, confrontando tais características com as tendências apontadas na literatura a respeito de e-learning.

Algumas características são consideradas importantes nas ferramentas de e-learning, principalmente o LMS, e se destacam nas ferramentas dos fornecedores de solução consideradas líderes pelo Relatório Gartner. Dois fatores apareceram com destaque entre os autores da literatura levantada e se confirmaram nas pesquisas: a) a integração com outros sistemas de Recursos Humanos contribui no gerenciamento dos funcionários, no desempenho destes e no plano de carreira dos mesmos e b) a inclusão de ferramentas e funcionalidades de redes sociais aumenta o interesse dos funcionários na utilização dos LMS e incentiva a transferência de conhecimento entre eles.

Além do que foi explanado, o maior interesse dos funcionários em utilizar as ferramentas, aumenta o interesse em compartilhar conhecimentos e obter novos conhecimentos aumentado também a receptividade dos funcionários envolvidos em relação aos novos conhecimentos. Além disso, contribui para incentivar o comportamento organizacional no que tange ao uso das informações e conhecimentos disponíveis melhorando a gestão do conhecimento da empresa.

A integração com outros sistemas melhora o controle e pode ajudar no gerenciamento do Capital Intelectual, principalmente, o capital humano, o que é evidenciado por muitos autores, como visto no referencial teórico, como um dos principais desafios das empresas hoje.

Outra tendência destacada na literatura e que está crescendo, conforme evidenciado na pesquisa, é a utilização do LMS em plataformas cloud computing (computação em nuvem). Dessa forma, a empresa líder Cornerstone OnDemand oferece sua solução LMS apenas como SaaS (Software as a service) e a e SAP Success Factors também já disponibiliza tal condição. No entanto, funcionalidades voltadas à gamificação não foram evidenciadas nas soluções LMS líderes analisadas.

A Computação em nuvem contribui também na retenção e documentação dos conhecimentos gerados e compartilhados, pois fornece um meio alternativo de retenção dos conhecimentos gerados, contribuindo para que tais conhecimentos permaneçam na organização, e, portanto, possam gerar acréscimos também ao capital intelectual da empresa.

Esta pesquisa apresenta como limitações o fato de a análise efetuada recair somente sobre quatro edições de relatórios de mercado, não obstante sejam as mais atuais. Outra limitação é o fato da análise ter sido feita considerando-se apenas as empresas citadas como líderes de mercado nos relatórios analisados.

Como sugestão de pesquisas complementares ou extensivas à presente análise, recomenda-se a verificação das soluções LMS analisadas na prática operacional das empresas. Ou seja, pesquisas futuras poderiam buscar a realização de estudo de casos em uma ou mais organizações, de modo a aprofundar o conhecimento acerca de como a solução LMS é utilizada pelas organizações, bem como a percepção dos usuários. Além disso, o estudo de casos aprofundaria a compreensão do que mais poderia ser feito para o melhor aproveitamento dos recursos disponibilizados pela solução LMS.

\section{REFERÊNCIAS}


BEZHOVSKI, Z; POORANI, S. The evolution of e-learning and new trends. Information and Knowledge Management, v. 6, n. 3, 2016.

CASTRO, C. M.; EBOLI, M. P. Universidade corporativa: gênese e questões críticas rumo à maturidade. Revista de Administração de Empresas, v. 53, p. 408-414, 2013. CLEMENTINO, A. Didática intercomunicativa em cursos online colaborativos. 238 f. Tese (Doutorado em Educação) - Universidade de São Paulo, São Paulo, 2008.

COHEN, E. Is the LMS dead? In: International Conference on Technology Supported Learning \& Training, $16^{\text {th }}$, Berlin, Alemanha, 2010. Proceedings... Berlin, 2010, p. 112.

CUELLAR, R. M.; AGUILAR, L. J. DACE, um modelo de aplicación de entornos virtuales de aprendizaje para la transferencia de conocimiento entre centros de formación profesional y empresas. Revista Iberoamericana de Educación. v. 60, p. 143-152, 2012.

FULLER, T. F. Beyond training: The impact of learning management system (LMS) on employee turnover. In: International Conference on e-Learning, 4th, Praga, 2013. Proceedings, Praga, 2013, p. 26-37.

GIL, A. C. Métodos e técnicas de pesquisa social. São Paulo: Atlas, 1999.

GODOY, A. S. Introdução à pesquisa qualitativa e suas possibilidades. Revista de Administração de Empresas, v. 35, n. 2, p. 57-63, 1995.

GARTNER. Magic quadrant for talent management suites. Stamford: Gartner, 2013. Magic quadrant for talent management suites. Stamford: Gartner, 2014. Magic quadrant for talent management suites. Stamford: Gartner, 2015.

Magic quadrant for talent management suites. Stamford: Gartner, 2017.

GURUNATH, R.; KUMAR, K. R. A., SaaS explosion leading to a new phase of a learning management system. Int J Cur Res Rev , v. 7, n. 22, Nov. 2015.

IGARASHI, W.; IGARASHI, D. C. C.; NAKAYAMA, N. K. Análise do alinhamento entre os elementos de aprendizagem organizacional, gestão do conhecimento e tecnologia da informação no contexto nacional e internacional. RENOTE - Revista Novas Tecnologias na Educação, v. 7, n. 1, p. 1-11, jul. 2009.

KANE, K.; COMBRE, J. R.; BERGE, Z. L. Tapping into social networking. Vine, v. 40, n. 1, p. 62-70, 2010.

OLIVEIRA, P. C.; CUNHA, C. J. C. A.; NAKAYAMA, M. K. Ambientes virtuais de aprendizagem e gestão da educação: uma revisão integrativa e agenda de pesquisa. JISTEM - Journal of Information Systems and Technology Management, v. 13, n. 2, p. 157-180, Maio/Ago. 2016.

PENA, R. F.; WALDMAN, F. B.; BERBAIN, N. M. S. P.; TEJADA, G. A.; CADIRANT, G. S.; PASSAGLIA, J.; CONTRERA, M. Implementación de los entornos virtuales de aprendizaje en cursos de capacitación docente. Revista Iberoamericana de Educación, v. 60, p. 117-128, 2012.

SILVA, J. C.; BRITO, A. V. Levantamento bibliográfico das publicações acadêmicocientíficas sobre análise de redes sociais aplicadas em e-learning. Novas Tecnologias na Educação, v. 12, n. 1, jul. 2014.

VENKATESWARAN, N. Strategies for adopting talent management issues in software companies. International Journal of Management, Economics and Social Sciences. v. 1, n. 2, p. 33-41, 2012.

YLMAZ, R. Knowledge sharing behaviors in e-learning community: Exploring the role of academic self-efficacy and sense of community. Computers in Human Behavior, v. 63, p. 373-382, 2016. 